\title{
A genetic algorithm to determine a production schedule under time-vary unit cost and shortages
}

\author{
Bing-Chang Ouyang ${ }^{1, a^{*}}$ and Liang-Bo Hsiao ${ }^{2, b}$ \\ ${ }^{12}$ Department of Business Administration \& Graduate School of Business and management, Vanung \\ University, Chungli, 32061, Taiwan, ROC \\ aouyang@vnu.edu.tw, bhs361543@gmail.com
}

\begin{abstract}
Keywords: Time-vary unit cost; Economic production quantity; Shortage; Genetic algorithm
Abstract. Considering a linear or exponential trend in unit production cost under a foreseeable time horizon, this study discusses the economic production quantity (EPQ) with shortages problem for a production system. A genetic algorithm (GA) with the chromosome of real number type to solve this problem is presented. Although, standard GA operators are used to generate new populations, the particular of this study is that we select two differentiate equations to develop a proposed production scheme. Then, compute the total cost with this production scheme as the fitness function to evaluate the populations. In this study, an explicit procedure to obtain the local optimal solution is provided and numerical examples to illustrate the proposed model are shown as well.
\end{abstract}

\section{Introduction}

The traditional economic production quantity (EPQ) model assumed a constant unit cost. Actually, the unit cost may vary with time. Recently, many businesses have confronted the price of merchandise fluctuating due to global economic events or technology innovation, which results in the risk of business trade increasing. Some outstanding enterprises even encountered heavy losses for neglecting the impact of this issue. During 2005-2007, emerging market economies, including the four BRIC (Brazil, Russia, India and China) countries, after witnessing a comparatively higher growth than rest of the world, caused the price of commodity increasing very steeply. In beginning 2008, the subprime mortgage problem in US triggered a worldwide financial crisis. The price of most commodities tumbled dramatically. According to International Monetary Fund [1] database for the price of commodity, the price of copper, a critical material of modern industry, was from US\$ 3241.9 to US\$ 8059.19 per ton (raised 162\%) during 2005M05-2006M05 due to BRIC flourishing. Conversely, the price of copper was from US\$ 8714.18 to US\$ 3770.88 per ton (dropped 57\%) during 2008M04-2009M03 owing to the US subprime crisis. The ASUS [2], the second largest of Taiwanese PC manufacturer, encountered its first quarterly loss since the company was founded in 1989 during the four quarter of 2008 for improper inventory policy and large inventory write-offs. Consequently, the traditional economic production quantity (EPQ) model assuming constant unit cost is no longer suitable in today's time-based competition. For advanced technology, Khouja \& Park [3] assumed that the unit price decreases exponentially and developed a closed-form approximate solution for a periodic EOQ model over a finite horizon. Later, Khouja \& Goyal [4] extended the unit cost to a linear or exponential function and presented a non-periodic EOQ policy. Under the same unit cost assumption, Ouyang and Rau [5] extended Khouja and Goyal [4] on products experiencing continuous decrease in unit cost to the case of a finite production capacity being inputted. None of the above contributions eliborate the EPQ with shortages. Applying genetic algorithms to the traditional economic lot size scheduling problem (ELSP), Khouja et al. [6] proposed a genetic algorithm to derive the solution. Hop and Tabucanon [7] encoded the timing of replenishment as a string of binary digits (a chromosome) and selected an adaptive genetic algorithm to handle the same problem.

\section{Assumptions and notation}

The assumptions for this proposed production system are as follows:

ü Unit cost is a linearly or exponentially continuous function.

$\ddot{u} \quad$ Demand is a constant over a finite time horizon and shortage is considered. 
$\ddot{u} \quad$ The production rate is always greater than or equal to the sum of the demand rate.

ü No stock is hold at beginning and end of the time horizon.

The following notation for our mathematical development is used.

$H \quad$ Finite planning horizon

$C_{s} \quad$ Unit shortage cost

$D$ Constant demand

$t_{i} \quad$ Finished time of the $i$ th production cycle

$P \quad$ Finite production rate

$Q_{i} \quad$ Production quantity of the $i$ th production cycle

$n \quad$ Number of production cycles

$f(t) \quad$ Unit production cost at time $t, f(t)=a-b t$ or $f(t)=a e^{-b t}$

$T_{i} \quad$ Time interval of the ith production cycle

$s_{i} \quad$ Completed shortage time of the $i$ th production cycle

$L_{i} \quad$ Maximum inventory level of the $i$ th production cycle

$h \quad$ Fraction of holding cost

$C_{r} \quad$ Production set up cost

$B_{i} \quad$ Maximum shortage of the $i$ th production cycle

TC Total cost

\section{The mathematical development}

Suppose a specified number of $n$ production cycles under the finite time horizon $H$. Fig. 1 depicts the inventory level of the $i$ th production cycle for this proposed model.

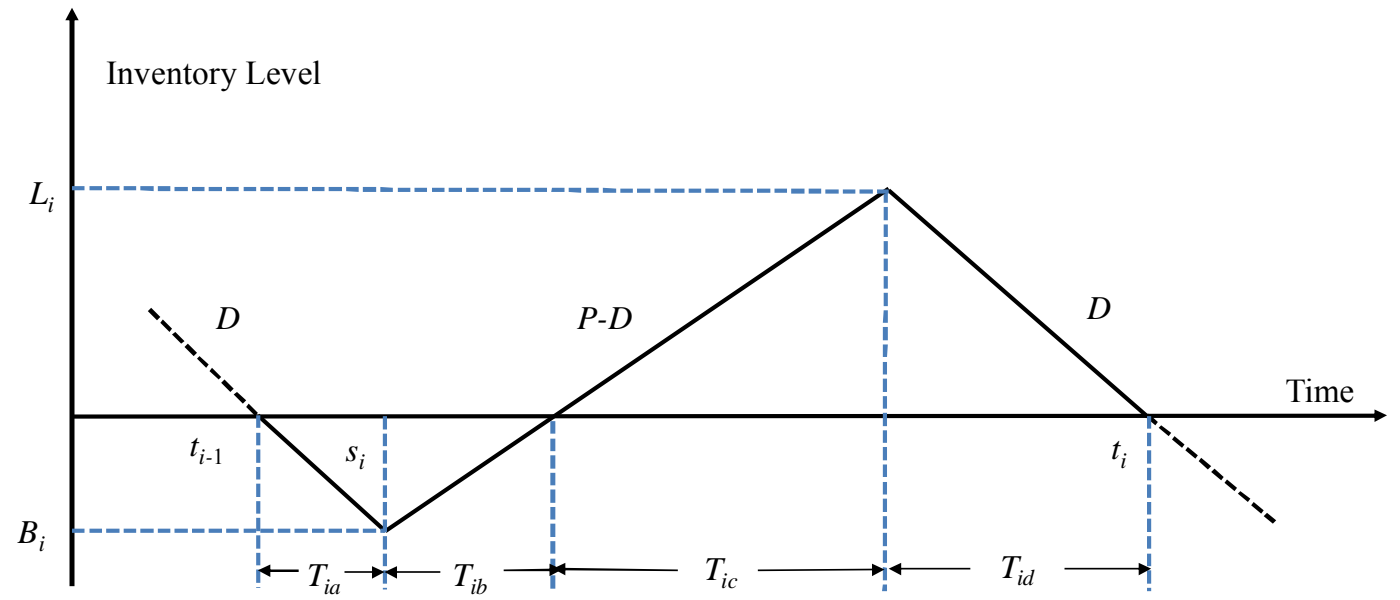

Figure 1. Inventory level for the $i$ th production cycle.

From Fig. 1, we hav $T_{i a}=\frac{B_{i}}{D}=s_{i}-t_{i-1}$ and $T_{i b}=\frac{B_{i}}{P-D}$. For $B_{i}=D\left(s_{i}-t_{i-1}\right)$ and $T_{i a}+T_{i b}=\frac{\left(s_{i}-t_{i-1}\right) P}{P-D}$, the time weight of shortage cost of the $i$ th production cycle $S C_{i}$ is as follows:

$$
S C_{i}=\frac{\left(s_{i}-t_{i-1}\right)^{2} P D}{2(P-D)} C_{s} \quad \text { for } \quad i=1,2, \ldots, n .
$$

Similarly, we know $\quad T_{i c}=\frac{L_{i}}{P-D} \quad$ and $\quad T_{i d}=\frac{L_{i}}{D} \quad$ and $\quad$ derive $T_{i c}+T_{i d}=\frac{P\left(t_{i}-s_{i}\right)-D\left(t_{i}-t_{i-1}\right)}{P-D}$ and $L_{i}=\frac{\left[P\left(t_{i}-s_{i}\right)-D\left(t_{i}-t_{i-1}\right)\right] D}{P}$ from Fig. 1, for $\left(t_{i}-s_{i}\right)=T_{i b}+T_{i c}+$ $T_{i d}$. Therefore, the time-weight of stockholding cost of the $i$ th production cycle is as follows:

$$
H C_{i}=\frac{\left[P\left(t_{i}-s_{i}\right)-D\left(t_{i}-t_{i-1}\right)\right]^{2} D}{2 P(P-D)} h f\left(s_{i}\right) \text { for } i=1,2, \ldots, n .
$$

The production quantity of this production cycle is

$Q_{i}=\left(t_{i}-t_{i-1}\right) D$.

Thus, the total cost of this proposed model $\operatorname{TC}\left(n,\left\{s_{i}\right\},\left\{t_{i}\right\}\right)$, including production set up cost, unit production cost, holding cost and shortage cost, is 


$$
\begin{aligned}
T C\left(n,\left\{t_{i}\right\},\left\{s_{i}\right\}\right) & =n C_{r}+\sum_{i=1}^{n}\left\{f\left(s_{i}\right)\left(t_{i}-t_{i-1}\right) D+\frac{\left[P\left(t_{i}-s_{i}\right)-D\left(t_{i}-t_{i-1}\right)\right]^{2} D}{2 P(P-D)} h f\left(s_{i}\right)\right. \\
& \left.+\frac{\left(s_{i}-t_{i-1}\right)^{2} P D}{2(P-D)} C_{s}\right\} \quad \text { for } i=1,2, \ldots, n
\end{aligned}
$$

Fix the number of production cycles $n$ and differentiate Eq. (4) with respect to $t_{i}$ and $s_{i}$. A necessary condition to acquire the optimal solution is that these two equations have to equal zero as follows:

$$
\begin{aligned}
\frac{\partial T C\left(n,\left\{t_{i}\right\},\left\{s_{i}\right\}\right)}{\partial s_{i}}= & f^{\prime}\left(s_{i}\right)\left(t_{i}-t_{i-1}\right) D+\frac{h f^{\prime}\left(s_{i}\right)\left[P\left(t_{i}-s_{i}\right)-D\left(t_{i}-t_{i-1}\right)\right]^{2} D}{2 P(P-D)} \\
& -\frac{h f\left(s_{i}\right)\left[P\left(t_{i}-s_{i}\right)-D\left(t_{i}-t_{i-1}\right)\right] D}{P-D}+\frac{\left(s_{i}-t_{i-1}\right) P D}{P-D} C_{s}=0
\end{aligned}
$$

for $i=1,2, \ldots, n$

$$
\begin{aligned}
\frac{\partial T C\left(n,\left\{t_{i}\right\},\left\{s_{i}\right\}\right)}{\partial t_{i}}=[ & \left.f\left(s_{i}\right)-f\left(s_{i+1}\right)\right] D+\frac{h f\left(s_{i}\right)\left[P\left(t_{i}-s_{i}\right)-D\left(t_{i}-t_{i-1}\right)\right] D}{P} \\
& +\frac{h f\left(s_{i+1}\right)\left[P\left(t_{i+1}-s_{i+1}\right)-D\left(t_{i+1}-t_{i}\right)\right] D^{2}}{P(P-D)} \\
& -\frac{\left(s_{i+1}-t_{i}\right) P D}{P-D} C_{s}=0, \text { for } i=1,2, \ldots, n-1 .
\end{aligned}
$$

In this problem, we have to determine the number of production cycles $n$ and production schedule $t_{i}$ and $s_{i}$, which is a complex nonlinear problem. Thus, we propose a genetic algorithm with the chromosome of real number type to seek an approximately optimal solution. According to Bellman's principle of optimization [8], once we find the correct $t_{1}$ and $s_{1}$ and the remaining production schedule $t_{2}$ to $t_{n}$ and $s_{2}$ to $s_{n}$ can be determine by Eqs. (5) and (6). Consequently, we only have to search $t_{1}$ and $s_{1}$ and neglect $n$. An explicit procedure to derive an approximate solution by this proposed genetic algorithm shows in the following:

Coding scheme:

We assume that the planning horizon is always less than one year. Therefore, randomly select a chromosome consisting of 20-bits for the first production cycle time $t_{1}$ under a reasonable range. For example, $t_{1}=0.125$ is represented by the bit string $0010,0000,0000,0000,0000$.

Fitness function:

With a trial values (chromosome) for the starting point $t_{1}$ in Eq. (5) to get $s_{1}$, replace $s_{1}$ and $s_{2}$ into Eq. (6) from Eq. (5) then obtain $t_{2}$. Repeatedly solve $t_{3}, t_{4}, \ldots \ldots, t_{n-1}, t_{n}$ until $t_{n-1}<H$ and $t_{n} \geq H$. Then, compute $T C\left(n,\left\{t_{i}\right\},\left\{s_{i}\right\}\right)$ where $t_{n}=H$ and $T C\left(n-1,\left\{t_{i}\right\},\left\{s_{i}\right\}\right)$ where $t_{n-1}=H$ from Eq. (4). Comparing these two total cost, we obtain the fitness function as follows:

The fitness function $\left\{\begin{array}{l}T C\left(n,\left\{t_{i}\right\},\left\{s_{i}\right\}\right) \quad \text { if } \quad T C\left(n,\left\{t_{i}\right\},\left\{s_{i}\right\}\right)<T C\left(n-1,\left\{t_{i}\right\},\left\{s_{i}\right\}\right) \\ T C\left(n-1,\left\{t_{i}\right\},\left\{s_{i}\right\}\right) \quad \text { if } \quad T C\left(n,\left\{t_{i}\right\},\left\{s_{i}\right\}\right)>T C\left(n-1,\left\{t_{i}\right\},\left\{s_{i}\right\}\right)\end{array}\right.$.

Genetic operators:

Three standard genetic operators are used, namely, reproduction, mutation and crossover.

Searching direction:

This GA searching direction is controlled by the self-adjustment rate of operators based on survivor off-springs' rates that are determined after each generation in order to the next search. Input parameters: 
$f(t)=40-5 t$ (Ex. 1), $f(t)=40+2 t\left(\right.$ Ex. 2), $P=16000, D=12000, h=0.08, \mathrm{C}_{s}=10, C_{r}=100, H=0.5$.

Parameters of genetic algorithm are population size $=100$, probability of mutation $=0.01$, probability of crossover $=0.7$, generation size $=100$, initial rate of crossover operation $=0.7$, initial rate of mutation operation $=0.25$, initial rate of reproduction operation $=0.05$ and stop condition.

Output: The local optimal production schedule and the total cost.

Step 1: Let $g=0$, where $g$ is the generation count.

Step 2: Generate initial population $P(g)$ randomly.

Step 3: Evaluate $P(g)$ to determine the best fitness value (best_fitness) and initialize the rates of operations. Repeat.

Step 4: Generate new population $P(g+1)$ using GA operators' rates from above offsprings.

Step 5: Evaluate $P(g+1)$ and determine number of survivors. Save the new best_fitness:

Step 6: Set $P(g)=P(g+1)$. Until a stopping criterion is reached.

Step 7: Compute the output based on the results.

Appling to our GA, the results of these two numerical examples are shown in Table 1.

Table 1. The results of numerical examples

\begin{tabular}{cccccccccc}
\hline$E x$. & $i$ & 1 & 2 & 3 & 4 & 5 & 6 & 7 & 8 \\
\hline & $s$ & 0.010 & 0.072 & 0.135 & 0.197 & 0.260 & 0.322 & 0.385 & 0.447 \\
$\mathbf{1}$ & $t$ & 0.062 & 0.125 & 0.187 & 0.250 & 0.312 & 0.375 & 0.437 & 0.500 \\
& $Q$ & 749.738 & 749.812 & 749.887 & 749.962 & 750.037 & 750.112 & 750.188 & 750.264 \\
\hline & $s$ & 0.006 & 0.255 & & & & & \\
2 & $t$ & 0.250 & 0.500 & & Total cost for example 1: 240120 & \\
& $Q$ & 2994.959 & 3005.041 & & Total cost for example 2: 241360 & \\
\hline
\end{tabular}

\section{Summary}

Foreseeing the unit production cost fluctuating under a planning horizon, this study presents a genetic algorithm to solve an EPQ with shortages problem. The particular of our proposed genetic algorithm is that we select Eqs. (5) and (6) to extend the production scheme for the fitness function. This algorithm has the potential applied to other complex problems. Thus, the further research direction will focus on various issues for a supply chain or production system.

\section{References}

[1] IMF, http://www.imf.org/external/np/res/commod/index.asp, available at (2013)

[2] Reuters, http://uk.reuters.com/article/2009/05/26/idUKTP3624762009 0526, available at (2014)

[3] M. Khouja \& S. Park, Optimal lot sizing under continuous price decrease. Omega, 31(6), (2003) 539-545.

[4] M. Khouja \& S. Goyal, Single item optimal lot sizing under continuous price decrease, International Journal of Production Economics, 102(1), (2006) 87-94.

[5] B. C. Ouyang, \& H. Rau, An economic production lot size for continuous decrease in production unit cost, Asia Pacific Journal of Operational Research, 25(5), (2008) 673-688.

[6] M. Khouja, Z. Mickalewicz, \& M. Wilmot, The use of genetic algorithms to solve the economic lot size scheduling problem, European Journal of Operation Research 110(2), (1998) 509-524.

[7] N.V. Hop, and M.T. Tabucanon, Adaptive Genetic Algorithm for Lot-sizing Problem with Selfadjustment Operation Rate, International Journal of Production Economics, 98(2),(2005) 129-135..

[8] R.E. Bellman, Dynamic Programming. Princeton University Press, Princeton, NJ, 1957. 